\title{
RESEARCH
}

Open Access

\section{Stakeholders' experiences of the public health research process: time to change the system?}

Yvonne Laird $^{1 *}$ (D), Jillian Manner ${ }^{2}$, Louise Baldwin ${ }^{3}$, Ruth Hunter ${ }^{4}$, John McAteer ${ }^{2}$, Sarah Rodgers ${ }^{5}$, Chloë Williamson ${ }^{6}$ and Ruth Jepson ${ }^{2}$

\begin{abstract}
Background: The importance of engaging stakeholders in the research process is well recognised. Whilst engagement is important, guidelines and practices vary for how stakeholders should be involved in research and how to facilitate effective collaborative relationships.

Methods: This study aimed to explore the perspectives and experiences of stakeholders involved in the policy and practice area of outdoor space and non-communicable disease prevention. Stakeholders interviewed included academics, practitioners, policy-makers, knowledge brokers and a funder.

Results: The findings suggest that stakeholders had positive experiences when engaged meaningfully in the research process, where research projects were carefully planned and managed with attention to context and culture, and where the research team was effective, respectful and communicative. These factors help to facilitate the translation of research into policy and practice. However, multiple challenges of collaborative research were identified which related to structural and systemic challenges, building and maintaining relationships, use and collection of data and information, cultural perceptions of research and research generation, and getting evidence into action. Participants felt that changing the funding system, exploring more collaborative research methodologies, improved research translation, and more effective collaborative relationships at all stages of the research process could address some of these challenges.

Conclusions: The findings highlight that, whilst stakeholder engagement in research was considered important, structural, cultural and individual practices impacted how this worked in practice. Identifying and testing solutions to address these challenges could improve synergies between research, policy, and practice and lead to the production of impactful research that reduces wastage of public funding, improves implementation of findings and ultimately improves public health outcomes.
\end{abstract}

Keywords: co-production, collaboration, interdisciplinary, action research, partnerships

\footnotetext{
* Correspondence: Yvonne.Laird@sydney.edu.au

'Sydney School of Public Health, Prevention Research Collaboration, Charles

Perkins Centre, University of Sydney, Sydney, Australia

Full list of author information is available at the end of the article
}

(c) The Author(s). 2020 Open Access This article is licensed under a Creative Commons Attribution 4.0 International License, which permits use, sharing, adaptation, distribution and reproduction in any medium or format, as long as you give appropriate credit to the original author(s) and the source, provide a link to the Creative Commons licence, and indicate if changes were made. The images or other third party material in this article are included in the article's Creative Commons licence, unless indicated otherwise in a credit line to the material. If material is not included in the article's Creative Commons licence and your intended use is not permitted by statutory regulation or exceeds the permitted use, you will need to obtain permission directly from the copyright holder. To view a copy of this licence, visit http://creativecommons.org/licenses/by/4.0/ The Creative Commons Public Domain Dedication waiver (http://creativecommons.org/publicdomain/zero/1.0/) applies to the data made available in this article, unless otherwise stated in a credit line to the data. 


\section{Background}

Non-communicable diseases (NCDs) are a major public health concern, responsible for almost $70 \%$ of all deaths worldwide [1]. Tobacco consumption, physical inactivity, alcohol misuse and unhealthy diet are risk factors for the rise in NCDs [2]. Despite efforts from policy-makers, practitioners and researchers to tackle NCDs, viable, sustainable, population-wide solutions have not yet been identified. Coupled with this, an estimated $85 \%$ of medical research expenditure is wasted through failure to disseminate, not publishing findings in an accessible format, not building on previous research, and failure to align research to policy, practitioner and public need $[3,4]$. If meaningful solutions to tackle NCDs are to be found, there is a need to identify new ways of working across research, policy and practice that reduces research wastage.

One such mechanism of addressing this lack of progress in NCD prevention is arguably through improving synergies between research, policy and practice systems. These synergies can potentially be enhanced through stakeholder engagement, which refers to the myriad of ways that stakeholders can be involved in the design, conduct and dissemination of research. Recent years have seen considerable growth in academic interest in collaborative engagement with policy and practice, in part due to the emphasis placed on impact by research quality assessments [5, 6]. Major funding bodies recommend the involvement of non-academics, which includes stakeholders not affiliated with academic institutions (e.g. members of the public, policy-makers, practitioners), in research projects and grant applications. Some funding bodies now offer dedicated funding streams for public engagement (e.g. the Wellcome Trust in the United Kingdom), increasing scholarly recognition of the potential for participatory research methods such as citizen science and community-based participatory research [7, 8]. Additionally, a number of national and international networks dedicated to stakeholder engagement in research have been established (e.g. https:// www.publicengagement.ac.uk, https://ecsa.citizen-science.net/, and http://coproductionscotland.org.uk).

Despite the growth of collaborative research, meaningful involvement of stakeholders in public health research, for example, as co-researchers, is not commonplace, with most research still predominantly conducted in academic institutions by researchers. In public health research, this lack of meaningful involvement seems at odds with principles of community engagement and intersectoral collaboration seen in public health practice. We argue there is a need to develop and embed (1) approaches to establishing and maintaining effective collaborative relationships with professionals in the research process and (2) approaches to meaningfully engage citizens in the research process such as citizen science and participatory action research. In this paper, we consider ways of enhancing the involvement and experiences of professional stakeholders in public health research.

Stakeholder engagement is important for public health science to ensure that planned or funded research is relevant and addresses key public health concerns for policy-makers, practitioners and the public. This engagement could involve identifying areas of need in local settings, prioritising research and providing input into the acceptability of research methods and tools. Such input increases the success of interventions, for example, by providing contextual information that can either impede or facilitate implementation. Research is more likely to be embedded in policy and practice when it is planned and conducted in conjunction with stakeholders, leading to enhanced research impact [9-11].

Challenges to stakeholder engagement include working with project partners who are geographically dispersed, management of divergent opinions across collaborators, power inequalities, competing priorities, less opportunities for face-to-face contact, partners who are less committed to stakeholder engagement, stakeholders who are not willing or interested in engaging in the research project, and striking a balance between the practical relevance of research and scientific merit [1214]. System level barriers, such as conflicting priorities and timescales between researchers, policy-makers and practitioners, funders priorities, and the academic reward system have also been identified [15-17]. Facilitators to collaborative working include supportive organisational principles (e.g. setting clear objectives and plans for stakeholder engagement), fostering shared values to research and stakeholder engagement, building trust, provision of training, and practices that consider the roles and responsibilities of stakeholders at each stage of the research process (e.g. considering how stakeholder input can be analysed) [12,18-21]. The quality of collaborative relationships, including the structure and process of collaborations, has also been identified as impacting partnership outcomes relating to good governance [14].

This research provides a starting point for developing effective collaborations with stakeholders and, whilst there is a growing body of research outlining the benefits of partnerships and collaborative methodological approaches (e.g. [13, 22-26]), there is an identified lack of guidance on forming and maintaining effective research partnerships [27], with existing evidence predominantly from the perspective of the academic. Understanding stakeholders' experiences of engaging in the research process could identify strategies aimed at better facilitating positive engagement. This study aimed to explore 
the perspectives and experiences of a range of professional stakeholders involved in generating or using research in public health. This paper will specifically focus on outdoor space and NCD prevention to provide a focus for participants to discuss, but the findings are likely to be generalisable to other research areas in public health. The objectives are to understand (1) the challenges and enablers of stakeholder engagement in the research process and use of research evidence; (2) experiences of stakeholder engagement in the research process; and (3) the ways in which the research process could better facilitate stakeholder engagement and the use of research evidence.

\section{Methods}

This qualitative interview study employed thematic analysis following the six-step approach outlined by Braun and Clarke [28] using a constructionist perspective. Thematic analysis enabled a rich theoretical insight into participants' experiences of stakeholder engagement. The study was conceived by a team of academics and non-academic partners, based on prior experiences of co-production and collaborative working. The project participants (both academic and nonacademic) shaped the direction of the research through providing informal feedback on the topic, the interview process and the initial findings. Ethical approval for this project was granted by the Usher Institute Research Ethics Committee at the University of Edinburgh (ethical approval code 1764). This study followed the procedures for reporting qualitative research outlined in the consolidated criteria for reporting qualitative research (COREQ) checklist [29].

\section{Sample and procedure}

A purposive sample of 33 policy-makers, researchers, practitioners, funders, consultants and directors of public health involved in generating or using research in outdoor space and NCD prevention in the United Kingdom were invited to take part via an email invitation. Of these, 21 agreed and 20 participated in a semi-structured interview following receipt of informed written consent. This included six in-person interviews, conducted in a quiet area in the participants' workplace, and 14 telephone interviews. Three researchers (YL, JM and CW), with training and experience in qualitative interviewing, conducted interviews between May and June 2018. Interviews were recorded using a digital audio recorder and lasted 22 to 54 minutes. The interviewers took detailed notes during interviews to aid reflection.

\section{Topic guide}

A topic guide was prepared with questions designed to elicit open responses. Questions were structured around
(1) participants' experiences of using and generating research, (2) enablers of using or generating research, (3) challenges of using or generating research, (4) perspectives on what needs to change to facilitate stakeholder engagement, and (5) experiences of collaborative research. Questions were modified based on whether the participant was a researcher, policy-maker, funder or practitioner. The topic guide was pilot tested and, following this, minor adaptions were made to improve clarity.

\section{Data analysis}

Interviews were transcribed verbatim, excluding identifiable information. Transcripts were read over carefully and checked for accuracy. The data were analysed inductively using the six-stage process of thematic analysis outlined by Braun and Clarke [28]; however, existing knowledge may also have influenced coding and themegeneration. Where possible, an iterative process was employed whereby interviews were conducted, transcribed and analysed, with the analysis informing subsequent data collection.

Coding was carried out by two researchers to facilitate reflection, discuss emerging findings and uncertainties, and to gain additional insights into the data. The first three transcripts were coded independently by two researchers. Following this, a coding framework was agreed between the researchers (Supplementary file 1). The coding framework was used as a guide for coding the remaining transcripts and was not prescriptive. Analysis was facilitated using NVivo 11 (QSR International). The coding of each transcript was cross-checked by another researcher (YL or JM), enabling additional reflexivity and the opportunity to discuss and explore uncertainties. The consistency of coding was checked between the initial and final transcripts. A total of 402 codes were initially identified and applied independently across the transcripts by two researchers. Codes that were similar were combined or grouped together to form overarching themes and sub-themes, with a final total of 60 codes. Any discrepancies were resolved through discussion with a third researcher. Data collection continued until the study authors were satisfied that the resulting data and analysis was sufficiently rich and adequately addressed the research questions. The final set of themes and sub-themes were discussed and agreed with the wider project team. A summary of the research findings was circulated to participants for comment. Any differences in opinion between academics and nonacademics are outlined in the findings.

\section{Results}

Participants included four senior academics, three policy-makers, nine practitioners, one funder, one 
consultant in public health, and two knowledge exchange experts $(n=20)$. Participants were based in universities, charities/non-profits, local and national government, the National Health Service (NHS) and government funded organisations in the United Kingdom. Some participants had multiple roles (e.g. responsibility for policy development and funding) and all participants (except the funder) had expertise in outdoor space and NCD prevention. Participants described factors that enabled engagement in the research process and use of research evidence, challenges and suggestions for change (Fig. 1). As demonstrated in Fig. 1, themes and sub-themes are not independent of each other and interact in a complex system.

\section{Enablers of stakeholder engagement in the research process}

Enabling factors included carefully planned and sufficiently resourced research, with an effective team. Participants noted that these criteria were central to the generation of high-quality, collaborative research.

Meaningful engagement with non-academics was considered to play a vital role to ensure that research is policy relevant, sensitive to local contexts, and that findings are implemented into practice. Participants felt that this engagement should be co-operative and should seek to understand and involve non-academics. Participants also noted that collaborative research should be mutually beneficial, potentially facilitating new lines of thinking, providing access to participants and data, and strengthening funding applications for academics whilst helping non-academics to gain traction and profile, influence research agendas, and improve their practice and ways of working.

"The more closely we can work with the research community the better able we are to be able to, on the one hand, to understand it ourselves. And on the other hand, to be able to support the research community in making sure that what they're producing is something that people outside of the research community are going to be able to understand. Whether that be the public or whether that be government departments." (Practitioner, participant 1)

There were mixed views as to how involved nonacademics felt they should be in the research process. Some wanted to engage regularly and contribute to funding bids, viewing research as an integral part of their role, whilst others felt they lacked capacity for research involvement. No clear methods for building collaborative relationships were identified; however, willingness to engage (in non-academics) and reputation (of academics) were mentioned by study participants.

\section{Challenges of engagement in the research process}

Challenges relating to building and maintaining relationships, data/information, getting evidence into action, culture and systems/structures were identified that made

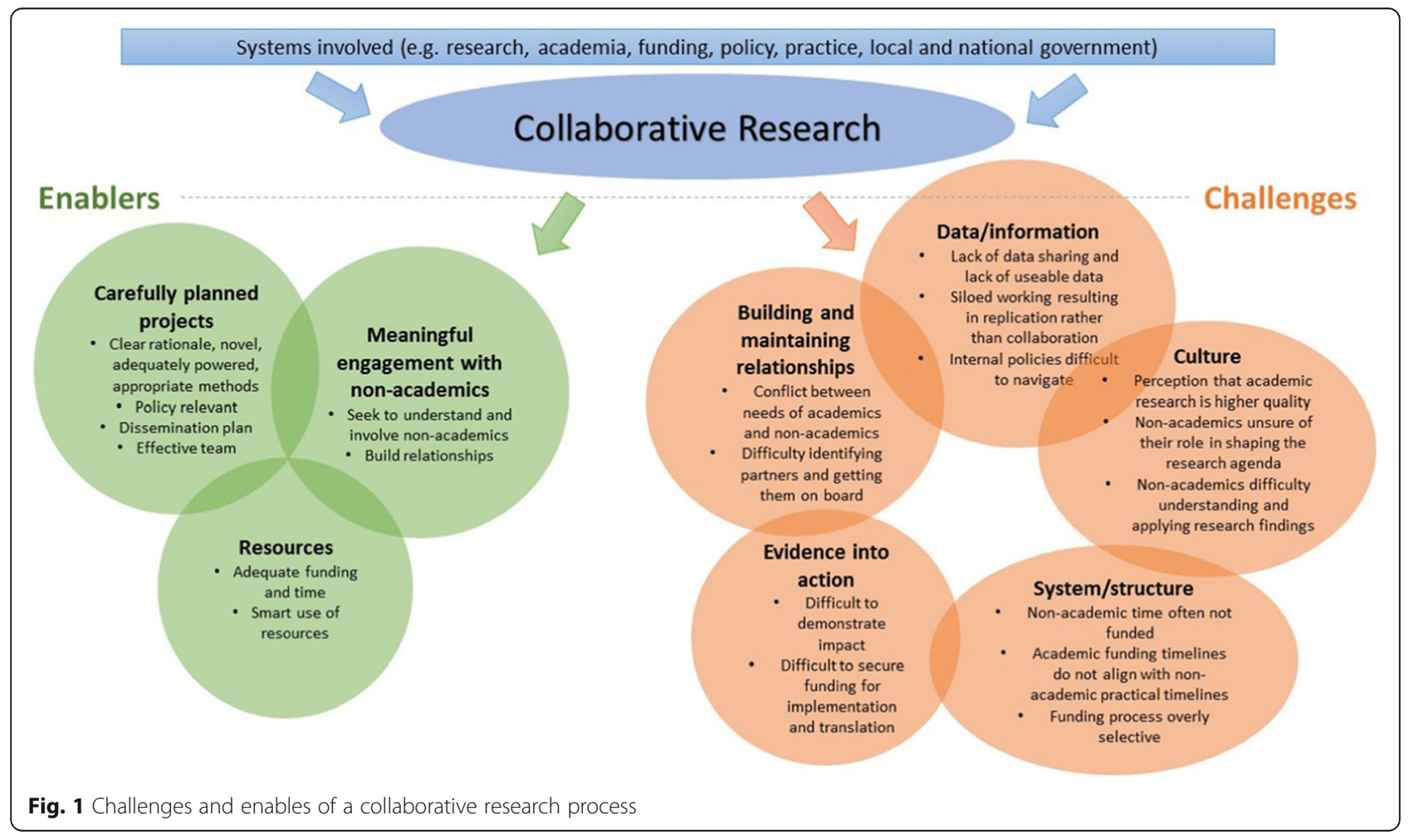


collaborative working and engaging in the research process difficult (Supplementary file 2).

\section{Building and maintaining relationships}

Establishing and building collaborative relationships was identified as time consuming and challenging for several participants, particularly those who lacked skills or confidence in networking. This included identifying and getting partners on board, connecting with people with shared interests and building multidisciplinary teams.

Maintaining relationships was also identified as a challenge. There was a recognition that partnerships do not always work for a range of reasons, for example, having incompatible disciplinary views. Academics found it difficult to manage relationships when the results were not what stakeholders expected. Conflicting priorities between practitioners and academics were also evident, for example, a practitioner may wish to deliver and evaluate multiple interventions in one setting, whereas an academic may prefer to deliver and evaluate only one. When research interests change and collaborators move roles, this also poses a challenge for maintaining working relationships. Finally, some interviewees noted that participants were sometimes involved in research without benefiting from their involvement or without an understanding of their needs. Identifying ways of engaging the public in research in mutually beneficial ways was identified as a priority moving forward.

"... often the people actually doing the work on the ground, have an initial, what's going on? You're getting in the way of my work because of course you have to get the funding, you have to get the baseline data in if it's an intervention and often that means you have to say 'yes, we want you to do the work but not yet', which can be maddening if you've got a community that wants something to happen, you've engaged with them, you've got the funding to actually do the intervention, you want to get on with it, not hold off whilst some academics fuss about getting the data before you do anything." (Academic, participant 2 )

\section{Data/information}

Participants described a lack of data sharing, lack of usable data, small sample sizes and poor-quality research as issues. In addition, duplication of research efforts due to a lack of communication and siloed working were observed. Ongoing research gaps were identified by non-academics that were not being addressed by academics due to a perceived lack of interest. Barriers to engaging in research, including a lack of knowledge of the research system, were identified by some non-academics. Similarly, others expressed difficulties in navigating internal policies with other organisations to collaborate on projects.
"People start scuttling back into silos... there's a lot of people doing stuff but it's just not being managed very well and the end result of that is that the community, I don't know, it doesn't serve anybody well, so if research can actually help to show efficacy of what really works and then people can pull together on a wider scale round that and say let's work together." (Practitioner, participant 4)

\section{Evidence into action}

Translating research into policy and practice was consistently identified by the participants as a challenge. Academics found it challenging to demonstrate impact. Similarly, translating research into practice was identified by non-academics as a challenge, particularly where funding for research translation or implementation was required. Some non-academics identified a disconnect between research and application and felt that research is not giving new insights into practice.

\begin{abstract}
"Often what you'll find is either a researcher or an academic will produce the classic piece of research and then think why is this not being acted on? Why has this not got its way into policy or practice? And why? It's because policy-makers or practitioners don't know about it, or because the timing's wrong. Or the flipside of it is the window of opportunity for a policy-maker will open and they'll be looking to evidence and knowledge base. If they haven't got that relationship and they don't know what evidence or research is out there or don't value it then they won't go looking for it. They won't be able to find it." (Practitioner, participant 5)
\end{abstract}

\section{Culture}

There was a perception amongst the participants that non-academic research is less rigorous than academic research, that some non-academics lack objectivity, and that academics are sceptical of non-academic research. Academic research was generally perceived to be of good quality, better resourced and of a high reputation.

There were uncertainties in relation to how much involvement non-academics should have in the research process and the role of non-academics in shaping the research agenda. Lack of time and funding were key barriers for non-academic involvement in research. Understanding and applying research findings to practice were further challenges for non-academics, with some feeling that they lacked research skills, which was a barrier to contributing to and using research.

The perception of the quality of research evidence was an identified challenge in relation to use of research evidence, with academics noting an ongoing perception in public health that randomised controlled trials are considered gold standard evidence. 
"... there needs to be a recognition in public health that natural experiments are sometimes the only possible and indeed the only appropriate ecologically valid way to understand relationships between use of the outdoor environment and health. Therefore, the weaknesses that inevitably in experimental design or associated with those, should not be seen as the research itself being of poor quality." (Academic, participant 2)

\section{System/structure}

Participants felt that funding processes can be overly selective and that changes in processes were difficult to keep up with. Time spent securing funding was an identified challenge and it was noted that academic funding timelines do not work with non-academic timelines. Additional funding challenges included funding not always being placed where it is most needed, funding favouritism (organisations preferring to fund specific researchers they know), government funding for research being used to justify pre-planned work, a lack of resources to commission or to fund work that needs to be done, lack of resources to fund the scale of research needed, or unwillingness to fund projects with long-term follow-up. Some participants also felt that academics were more inclined to submit 'safer' projects, which were less reflective of stakeholder need, due to the funding process and job security. Academic involvement in projects was felt to be expensive, particularly for government or not-for-profitfunded projects. Some non-academics wanted to influence research agendas and the nature of research call documents but were unsure about how they could do this. Finally, there was a recurring concern amongst nonacademics that time for their involvement in research projects is often not funded, yet non-academics are expected to make large time commitments to communicate and collaborate with researchers to achieve project goals. In contrast, one academic felt that non-academics were happy to be involved in research without their time being funded. "[We] never saw a penny of the research funding ... it was really difficult for us in terms of just creating the time and the space to engage. But we did see a value in that" (Practitioner, participant 1).

Non-academics noted that the academic research process was slow and felt frustrated about having to wait for publication to see results and the time it takes to obtain research approvals. Competing tasks or projects were felt to compound this. This presented a challenge for applying research findings or committing to engaging in research when stakeholder priorities are likely to change before funded research projects could begin.

Getting evidence into action was identified as a challenge and, equally, it was felt that research is not always informed by policy and practice or useful for this audience. A rapidly changing political context, in contrast to academic timescales, was also found to present a challenge for the generation of impactful and usable research. A need for skills to bridge the gap between academics and non-academics was identified to ensure that research is informed by policy and practice and vice versa. Finally, one participant expressed concerns that the government suppressed unfavourable research findings, where the findings were deemed to conflict with current political interest.

\section{Suggestions for change}

Suggestions were put forward to address the challenges identified. These included changes to the funding system, methodologies, evidence into policy and practice, and to methods for collaborative working.

\section{The funding system}

A proposal was made that funders should actively and regularly consider ways to improve the funding system, for example, by funding non-academic involvement in research and involving non-academics in shaping the research that is commissioned.

"... it feels tremendously farfetched, but if ever there was a mechanism whereby [non-academic] organisations ... could be supported centrally through the research councils or research funders, to almost give us a mandate to go and work with the research community on projects. So almost a ... I don't know what you'd call it really, but an allocation that enabled us to engage with bits of the research community would be an amazing solution to it, I think. I've never had that conversation with the research councils. But if they said right, there's enough funding to have a dedicated person who just works with the research community, then we could immediately go and find 20 projects that would really welcome our involvement at no cost to them. That we could really support and work with and hopefully improve the research outcomes from those projects." (Practitioner, participant 1)

\section{Methodologies}

Suggestions for changing research methodologies to support collaborative working and use of research included measuring relevant outcomes at appropriate time points, preparing contingency plans and using research approaches that engage meaningfully with participants. Citizen science and community action research were proposed as potential methods of facilitating empowering community engagement.

"Working with community groups, with research mentors, to help them identify their own research questions and then to develop and design research programmes 
to answer the questions themselves ... that has a huge power because it puts the hands of the research question and then taking the research in the community, so it's something that they are doing themselves and doing with research partners, rather than being the subject of research and research being done to them." (Practitioner, participant 9)

\section{Evidence into policy and practice}

Suggestions to enhance the use of evidence in policy and practice included obtaining a clearer picture of what government needs to inform policy, training postgraduate students on creating impactful research, effective dissemination of research that is engaging and understandable to non-academics, developing skills in translation of research findings, and roles that bridge the gap between academic and non-academics such as knowledge-exchange brokers.

\section{Better collaboration between academics and non- academics}

Several participants wanted to see more meaningful collaboration between academics and non-academics. Participants felt that this could enable non-academics to shape research priorities and designs, enable data sharing and find out about new research. However, as previously noted, some participants were satisfied with their level of involvement or felt that involvement in research was not part of their job. Skill development in forming collaborative relationships and networking was identified as a need amongst some participants.

\section{Discussion}

NCDs are a growing public health concern and, given the estimated high levels of research wastage and lack of viable solutions to tackle NCDs, there is a need for honest reflections on the public health research process. There is also a need to recognise the research system as a complex system, which interacts with and is influenced by multiple other systems, for example, funding, local and national government, and education systems. Framing and viewing the research system using systems thinking can help to identify and develop sustainable approaches to improving the research process [30]. This study qualitatively explored stakeholders' experiences of engaging in the research process in outdoor space and NCD prevention. Enablers, challenges and suggestions for improvement to the research process were identified. The findings build on previous research on partnerships [14] and provide a starting point to enhance the research-policy-practice relationship using systems thinking, ultimately to tackle the growing burden of NCDs.

Carefully planned and resourced research, including team members with appropriate skills, was found to facilitate stakeholder engagement in the research process. Consistent with previous research, carefully planned and managed research with attention to context and culture, and where the research team was effective, respective and communicative, was found to enable the use of research evidence and facilitate positive experiences of engagement in the research process [31, 32]. Embedding collaborative research into routine practice could be enabled through policy changes at the research and funding level. For example, all research (where appropriate) at the Scottish Collaboration for Public Health Research and Policy, University of Edinburgh, involves collaborations with policy-makers, practitioners or community members with the collective goal of improving public health. This contributes to building mutually beneficial relationships, where collaborators and researchers can propose research questions, conduct research and implement findings. Considering the feasibility of introducing policies aimed at enabling collaborative research practices, whilst maintaining researcher objectivity and an ability to produce revolutionary scientific advances, has the potential to address the challenges identified in this study across the identified sub-themes. Such strategies could develop the learning of both stakeholders and researchers, assist with the translation of research findings, and lead to the production of research that better meets the needs of community members [12, 31].

Policies and strategies aimed at increasing collaborative research are unlikely alone to transform stakeholder engagement in the research process and it is important that any policies in relation to collaborative working sit within a broader suite of strategies to address the challenges identified. Challenges ranged from factors that could be relatively straightforward to address (e.g. collaborators moving roles) and public health capacitybuilding, to complex, systemic factors required to better align research, policy and practice systems, which adds to previous research findings [33, 34]. Systemic changes would arguably be more difficult to address (e.g. the nature of research funding and the academic system). It is important that any solutions address identified barriers for non-academic participation, such as costing the time of non-academics in research funding bids for collaborative projects where appropriate. Some potential solutions to some of these challenges, based on our own experiences of the research system, are outlined in Table 1. Implementing and evaluating these potential solutions and identifying and piloting additional strategies to address the identified challenges using systems thinking could provide further insight to improve the researchpolicy-practice relationship and make the research system more accessible to non-academics.

Whilst in this paper we have discussed non-academic partners as practitioners, policy-makers and other 


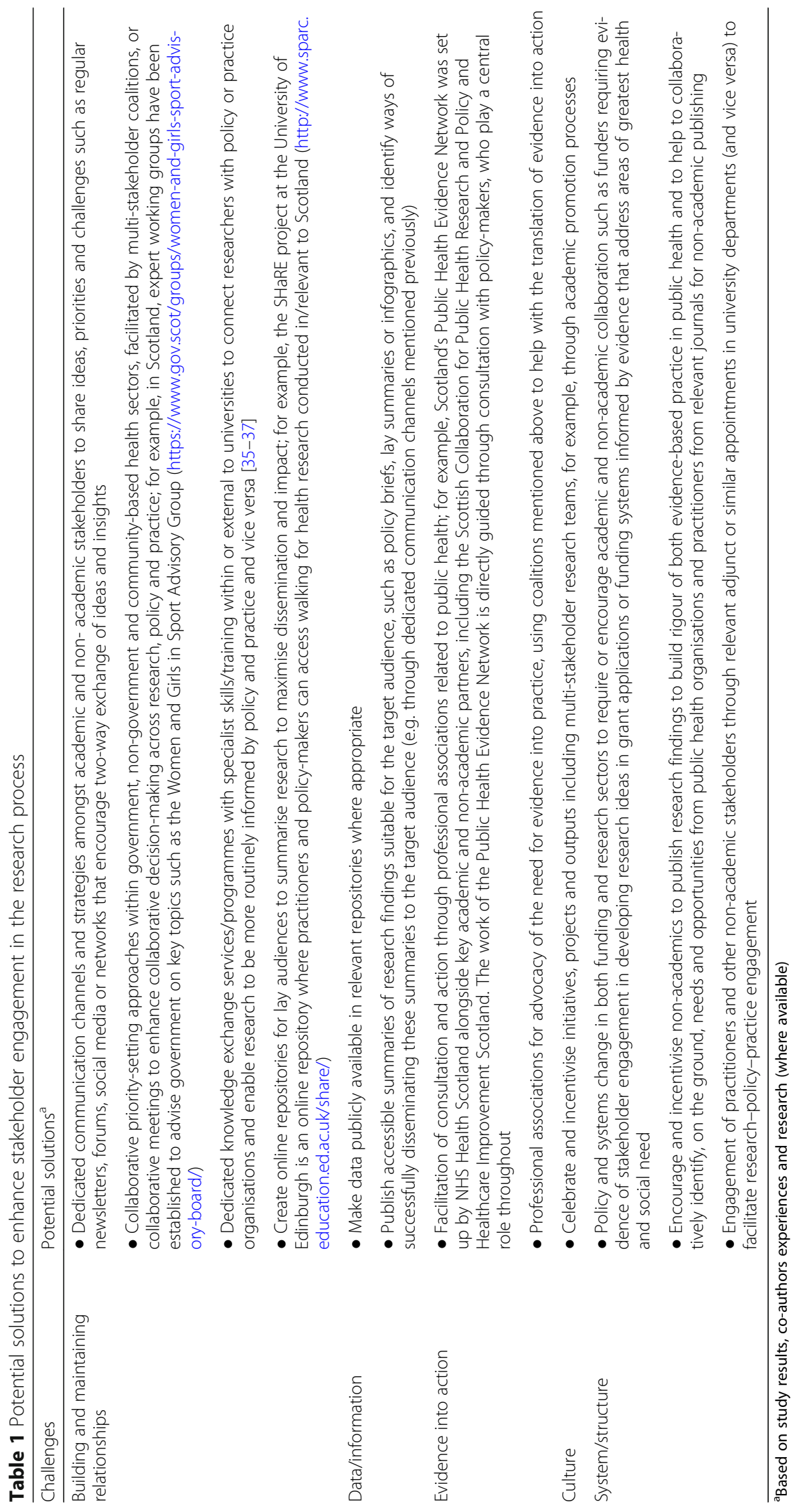


professionals involved in public health, naturally, the community is also an integral stakeholder; this has been well noted in previous reports [19, 38] and is further supported by these findings. Our research suggests a need for a wider discussion on how to engage the public in research in ways that are meaningful and empowering, enabling members of the public to be involved in the strategic direction of research as well as the conduct, analysis and implementation of findings. Citizen-led approaches, such as citizen science, may enable the public to develop skills and use these skills and the data generated to improve their local communities. The development of these and other citizen-led research approaches has been suggested to have the potential to transform public health science and policy-making [8] and should be an ongoing discussion for the research, policy and practice community.

\section{Strengths and limitations}

A broad range of perspectives and experiences, including representatives from policy, practice, research, knowledge brokers and funders, were included to outline the challenges and enablers to stakeholder engagement in the research process. This contributed to rich data addressing the research questions. Whilst a broad range of perspectives were included, findings are limited to a relatively small sample of professionals $(n=20)$ from a broad range of organisations with interests in outdoor space and NCD prevention in a United Kingdom context. Thus, the findings may not be transferable to other countries or disciplines where differences in collaborative relationships or the research process may exist. Including perspectives from members of the public and academic journal editorial staff could have provided additional insights.

\section{Conclusions}

This study identified a range of facilitators and challenges in relation to stakeholder engagement in research across multiple levels. Challenges related to forming and maintaining collaborative relationships, accessibility of data/information, getting evidence into action, culture, and systemic and structural challenges. The findings of this study add to existing literature on the importance of collaborative research for research impact; however, the identified barriers highlight structural, cultural and individual barriers that need to be addressed to enable effective collaborative relationships between research, policy and practice. Developing and piloting approaches in collaboration with stakeholders to address the identified challenges of stakeholder engagement using systems thinking could contribute to positively changing the research system and how research, policy and practice intertwines, and ultimately improve research outcomes and reduce NCDs.

\section{Supplementary information}

Supplementary information accompanies this paper at https://doi.org/10. 1186/s12961-020-00599-5.

Additional file 1. Fishbone diagram.

Additional file 2: Supplementary file A. Basic coding framework.

Abbreviation

NCDs: non-communicable diseases

\section{Acknowledgements}

The authors would like to thank their research partners who have shaped their perspectives on collaborative research practices and the participants who took part in this research.

Authors' contributions

$\mathrm{RJ}$ and JMC conceived the study; JMC, RJ, YL and JM planned the study; YL, JM and CW collected and analysed the data; YL initially drafted the manuscript; all authors read, contributed to and approved the final version.

Funding

This work was funded by the Medical Research Council, Grant number RA2497.

\section{Availability of data and materials}

The datasets used and/or analysed during the current study are available from the corresponding author on reasonable request.

\section{Ethics approval and consent to participate}

Ethical approval for this project was granted by the Usher Institute Research Ethics Committee at the University of Edinburgh (ethical approval code 1764). All participants provided written informed consent.

Consent for publication

Not applicable.

\section{Competing interests}

All authors are active researchers in public health. The authors have no other competing interests to declare.

\section{Author details}

${ }^{1}$ Sydney School of Public Health, Prevention Research Collaboration, Charles Perkins Centre, University of Sydney, Sydney, Australia. ${ }^{2}$ Scottish Collaboration for Public Health Research and Policy, School of Health in Social Science, University of Edinburgh, Edinburgh, United Kingdom. ${ }^{3}$ School of Public Health and Social Work, Queensland University of Technology (QUT), Institute of Health and Biomedical Innovation, Brisbane, Australia. ${ }^{4}$ Centre for Public Health, Queen's University Belfast, Belfast, United Kingdom. ${ }^{5}$ Department of Public Health and Policy, University of Liverpool, Liverpool, United Kingdom. ${ }^{6}$ Physical Activity for Health Research Centre, Institute of Sport, Physical Education and Health Sciences, Moray House School of Education and Sport, University of Edinburgh, Edinburgh, United Kingdom.

Received: 22 October 2019 Accepted: 3 July 2020

Published online: 18 July 2020

\section{References}

1. World Health Organization. Global Status Report on Noncommunicable Diseases. Geneva: WHO; 2014.

2. Forouzanfar MH, Alexander $L$, Anderson HR, Bachman VF, Biryukov S, Brauer $\mathrm{M}$, et al. Global, regional, and national comparative risk assessment of 79 behavioural, environmental and occupational, and metabolic risks or clusters of risks in 188 countries, 1990-2013: a systematic analysis for the Global Burden of Disease Study 2013. Lancet. 2015;386(10010):2287-323.

3. Chalmers I, Glasziou P. Avoidable waste in the production and reporting of research evidence. Lancet. 2009;374(9683):86-9.

4. Wolfenden $L$, Ziersch A, Robinson P, Lowe J, Wiggers J. Reducing research waste and improving research impact. Aust N Z J Public Health. 2015;39(4): 303-4. 
5. Research Excellence Framework. What is the REF? 2019. https://www.ref.ac uk/about/what-is-the-ref/. Accessed 21 Aug 2019.

6. Australian Research Council. Excellence in Research for Australia. 2019. https://www.arc.gov.au/excellence-research-australia. Accessed 21 Aug 2019.

7. Baum F, MacDougall C, Smith D. Participatory action research. J Epidemiol Community Health. 2006;60(10):854.

8. Rowbotham S, McKinnon M, Leach J, Lamberts R, Hawe P. Does citizen science have the capacity to transform population health science? Crit Public Health. 2019;29(1):118-28.

9. Kok MO, Gyapong JO, Wolffers I, Ofori-Adjei D, Ruitenberg J. Which health research gets used and why? An empirical analysis of 30 cases. Health Res Policy Syst. 2016;14:36.

10. Innvaer S, Vist G, Trommald M, Oxman A. Health policy-makers' perceptions of their use of evidence: a systematic review. J Health Serv Res Policy. 2002; 7(4):239-44.

11. Orton L, Lloyd-Williams F, Taylor-Robinson D, O'Flaherty M, Capewell S. The use of research evidence in public health decision making processes: systematic review. PLoS One. 2011;6(7):e21704

12. Boaz A, Hanney S, Borst R, O'Shea A, Kok M. How to engage stakeholders in research: design principles to support improvement. Health Res Policy Syst. 2018;16(1):60.

13. Stokols D. Toward a science of transdisciplinary action research. Am J Community Psychol. 2006;38(1-2):63-77.

14. Brinkerhoff J. Partnership as a Means to Good Governance: Toward an Evaluation Framework. Cheltenham: Edward Elgar Publishers; 2008. p. 68-89.

15. van de Goor I, Hämäläinen R-M, Syed A, Juel Lau C, Sandu P, Spitters H, et al. Determinants of evidence use in public health policy making: results from a study across six EU countries. Health Policy. 2017;121(3):273-81.

16. Green LW, Glasgow RE, Atkins D, Stange K. Making evidence from research more relevant, useful, and actionable in policy, program planning, and practice: slips "twixt cup and lip". Am J Prev Med. 2009;37(6 Suppl. 1):S187S91.

17. Moore JB, Maddock JE, Brownson RC. The role of dissemination in promotion and tenure for public health. J Public Health Manag Pract. 2018; 24(1):1-3

18. Giles-Corti B, Sallis JF, Sugiyama T, Frank LD, Lowe M, Owen N. Translating active living research into policy and practice: one important pathway to chronic disease prevention. J Public Health Policy. 2015;36(2):231-43.

19. Jagosh J, Bush PL, Salsberg J, Macaulay AC, Greenhalgh T, Wong G, et al. A realist evaluation of community-based participatory research: partnership synergy, trust building and related ripple effects. BMC Public Health. 2015; 15:725.

20. Pinto RM. What makes or breaks provider-researcher collaborations in HIV research? A mixed method analysis of providers' willingness to partner. Health Educ Behav. 2013;40(2):223-30.

21. Salsberg J, Parry D, Pluye P, Macridis S, Herbert CP, Macaulay AC. Successful Strategies to Engage Research Partners for Translating Evidence into Action in Community Health: A Critical Review. J Environ Public Health. 2015;2015: 15.

22. Hawkins J, Madden K, Fletcher A, Midgley L, Grant A, Cox G, et al. Development of a framework for the co-production and prototyping of public health interventions. BMC Public Health. 2017;17:689.

23. Bonney R, Cooper CB, Dickinson J, Kelling S, Phillips T, Rosenberg KV, et al. Citizen science: a developing tool for expanding science knowledge and scientific literacy. BioScience. 2009;59(11):977-84.

24. Giles-Corti B, Whitzman C. Active living research: partnerships that count. Health Place. 2012;18(1):118-20.

25. Wight $D$, Wimbush $E$, Jepson $R$, Doi L. Six steps in quality intervention development (6SQuID). J Epidemiol Community Health. 2016;70(5):520-5.

26. Craig P, Dieppe P, Macintyre S, Michie S, Nazareth I, Petticrew M. Developing and evaluating complex interventions: the new Medical Research Council guidance. BMJ. 2008;337:a1655.

27. de Moissac D, Bowen S, Botting I, Graham ID, MacLeod M, Harlos K, et al. Evidence of commitment to research partnerships? Results of two web reviews. Health Res Policy Syst. 2019;17:73.

28. Braun V, Clarke V. Using thematic analysis in psychology. Qual Res Psychol. 2006;3(2):77-101.

29. Tong A, Sainsbury P, Craig J. Consolidated criteria for reporting qualitative research (COREQ): a 32-item checklist for interviews and focus groups. Int J Qual Health Care. 2007;19(6):349-57.
30. Rutter H, Savona N, Glonti K, Bibby J, Cummins S, Finegood DT, et al. The need for a complex systems model of evidence for public health. Lancet. 2017;390(10112):2602-4.

31. Jolibert C, Wesselink $A$. Research impacts and impact on research in biodiversity conservation: the influence of stakeholder engagement. Environ Sci Policy. 2012;22:100-11.

32. Tricco AC, Cardoso R, Thomas SM, Motiwala S, Sullivan S, Kealey MR, et al. Barriers and facilitators to uptake of systematic reviews by policy makers and health care managers: a scoping review. Implement Sci. 2016;11:4.

33. Taylor-Robinson DC, Lloyd-Williams F, Orton L, Moonan M, O'Flaherty M, Capewell S. Barriers to partnership working in public health: a qualitative study. PLoS One. 2012;7(1):e29536.

34. Brownson RC, Fielding JE, Green LW. Building capacity for evidence-based public health: reconciling the pulls of practice and the push of research. Annu Rev Public Health. 2018;39(1):27-53.

35. Moore G, Redman S, D'Este C, Makkar S, Turner T. Does knowledge brokering improve the quality of rapid review proposals? A before and after study. Syst Rev. 2017;6:23.

36. Elueze IN. Evaluating the effectiveness of knowledge brokering in health research: a systematised review with some bibliometric information. Health Inform Libr J. 2015;32(3):168-81.

37. Bornbaum CC, Kornas K, Peirson L, Rosella LC. Exploring the function and effectiveness of knowledge brokers as facilitators of knowledge translation in health-related settings: a systematic review and thematic analysis. Implement Sci. 2015;10:162.

38. Goodman MS, Sanders Thompson VL, Johnson CA, Gennarelli R, Drake BF, Bajwa $P$, et al. Evaluating community engagement in research: quantitative measure development. J Community Psychol. 2017;45(1):17-32

\section{Publisher's Note}

Springer Nature remains neutral with regard to jurisdictional claims in published maps and institutional affiliations.

Ready to submit your research? Choose BMC and benefit from

- fast, convenient online submission

- thorough peer review by experienced researchers in your field

- rapid publication on acceptance

- support for research data, including large and complex data types

- gold Open Access which fosters wider collaboration and increased citations

- maximum visibility for your research: over $100 \mathrm{M}$ website views per year

At BMC, research is always in progress.

Learn more biomedcentral.com/submissions 